\title{
Comparison of Data with Multiple Degrees of Freedom Utilizing the Feature Selective Validation (FSV) Method
}

\author{
Gang Zhang, Alistair Duffy, Fellow, IEEE, Antonio Orlandi, Fellow IEEE, Danilo Di Febo, Lixin Wang, \\ Hugh Sasse
}

\begin{abstract}
The Feature Selective Validation method has been shown to provide results that are in broad agreement with the visual assessment of a group of engineers for line, 1-dimensional, data. An implementation using 2-dimensional Fourier transforms and derivatives has been available for some years, but verification of the performance has been difficult to obtain. Further, that approach does not naturally scale well for 3-dimensional and higher degrees of freedom, particularly if there are sizable differences in the number of points in the different directions. This paper describes an approach based on repeated 1-dimensional FSV analyses that overcomes those challenges. The ability of the 2-dimensional case to mirror user perceptions is demonstrated using the LIVE database. Its extension to $n$-dimensions is also described and includes a suggestion for weighting the algorithm based on the number of data points in a given 'direction'.
\end{abstract}

Index Terms-Feature Selective Validation, Validation, Modeling, Measurement, Comparison

\section{INTRODUCTION}

$\mathrm{E}$ lectromagnetic Compatibility (EMC) and Signal Integrity/Power Integrity (SI/PI) data with multiple degrees of freedom (or dimensions) is becoming commonplace. This can arise in both simulation-based and measurement-based environments [1] [2], e.g. fields on a plane, in a volume, surface current or data for every wire in a loom. Determining a level of agreement between two or more dimensional data sets is a challenging, if not impossible, task, particularly if that comparison information needs to be resolvable to a measure that can be readily communicated within or beyond project teams.

For the comparison of one dimensional (1D) data, the Feature Selective Validation (FSV) method was developed [3]-[5] and chosen as reference technique by the IEEE Standard P1597 [6] [7], which benefits from the ability to mirror the

Manuscript received February 5th, 2016. This work was supported by the National Natural Science Foundational of China under Grant No. 51507041.

Gang Zhang and Lixin Wang are with Harbin Institute of Technology, Harbin, China (phone: +86-451-86403705; e-mail: 09B906023@hit.edu.cn; wlx@hit.edu.cn). Alistair Duffy and Hugh Sasse are with De Montfort University, Leicester, UK (e-mail: apd@dmu.ac.uk; hgs@dmu.ac.uk).

Danilo Di Febo and Antonio Orlandi are with the UAq EMC Laboratory, University of L’Aquila, Italy (e-mail: danilodifebo@gmail.com; antonio.orlandi@univaq.it). human decision making process in the visual assessment of line-data comparisons. Specifically, the examination of the data by engineers usually consists of looking at the general trends and the details as separate but connected processes. The FSV method accounts for this fact by filtering out the DC, Low- and High-frequency components from the original data. The data is first transformed into the frequency domain, the lowest five points are separated out as DC, and the rest divided such that $40 \%$ of the energy in the graph is in the low frequency region, the rest in the high frequency region. Then the components are transformed back to the original domain. The Amplitude Difference Measure (ADM) and Feature Difference Measure (FDM) are performed based on these components to reflect the trend and feature differences between data sets, respectively. The Global Difference Measure (GDM) is a combination of the ADM and FDM.

The outputs of the FSV method are presented in different layers. $x D M i$ ( $x$ is $A, F$ or $G$ ) is a point-by-point indicator, the synthetic figure of merits of the comparison, $x D M$ tot ( $x$ is $A, F$ or $G$ ), is obtained by taking the average of $x D M i$. Furthermore, the aforementioned quantitative results can be converted to natural language descriptors in a six level scale: Excellent, Very Good, Good, Fair, Poor, Very Poor [8]. Based on this, the $x$ DMc ( $x$ is $A, F$ or $G$ ) is obtained to show the proportion of the point-by-point results that falls into the six natural language descriptor categories. It has been shown that the GDMc agrees with the overall group opinion [4] [9]. Several enhancements to the method have been proposed [10-12], in particular the introduction of the grade-spread diagram in [13]. Further details of the FSV method can be found in [6].

With the need to develop a two or higher dimensional (2D or $n \mathrm{D})$ data quantitative validation method, the approach extending 1D FSV to allow the validation of 2D data has been studied. The implementation in [5] treated the $2 \mathrm{D}$ data as a folded 1D data and, therefore, the filters were only first order representations of $2 \mathrm{D}$ filters. This approach is simple and easy to implement, but does not fully allow for $n \mathrm{D}$ data with features that differ in all directions, because the converted $1 \mathrm{D}$ data is assembled in one direction.

The strategy applied in [14] (labeled as 2D-FSV-UAq) followed the general implementation of the 1D FSV, which was performed by replacing 1D Fourier Transform with a 2D Fourier Transform to the data and to window the transformed 
data to separate out the DC, Low- and High-frequency components in 2D. Using this approach, the obtained results could be rated using the standard FSV interpretation scale. Reference [15] takes a similar approach. Nevertheless, both the approaches do not naturally scale well for 3D and higher degrees of freedom, particularly if there are sizable differences in the number of points in different directions. Also the computation of Fourier Transform becomes more and more resource intensive with the rise in the level of dimensionality.

As the central principle followed by FSV is to mirror the evaluation process of a group of experts, higher levels of dimensionality provide substantial challenges for calibration. To date, neither of the aforementioned 2D FSV approaches has been substantially verified.

This paper aims to develop an $n \mathrm{D}$ FSV approach by directly and repeatedly applying the 1D FSV to the data with multiple degrees of freedom: this method is labelled as 2D-FSV-HIT in the following sections and should be readily extendible to $n \mathrm{D}$. Further, all the improvements and enhancements to 1D FSV can be directly applied to $n \mathrm{D}$ without being "re-cast" in some way [16] [17]. It is also an approach that can allow very different numbers of data points in different directions. This paper will focus discussions on these issues. In section II, a 2D FSV approach based on 1D FSV is introduced. The performance of the proposed approach is examined and compared with the existing 2D FSV method in Section III. An example of implementation is analyzed in Section IV. The conclusion is drawn in Section V.

\section{THE 2D-FSV BASED ON 1D-FSV}

Generally, the approach is to repeatedly use 1D FSV on each line of data and then each column of data. To get the combined $x D M i$, the weighted root square at any one point is taken, which will keep FSV in the 'normal' range, as referred to 1D FSV, irrespective of the number of degrees of freedom. The $x D M t o t$ data is obtained in the same way as for 1D FSV. The general procedure of the 2D-FSV-HIT is as follows.

It should be first ensured that the 2D data sets under comparison have the same number of data points located at the same positions on the independent $(x, y)$ axes. If not, we need to obtain the overlap surface window and interpolate the data in the overlap region to ensure coincident pairs of data points.

If the input 2D data is $I 1$ and $I 2$ with the same size of $M^{*} N$, they are compared by:

(1) Treat each row data, $I_{\{H 1, H 2\}}(y)(y=1,2,3, \cdots, N)$, and each column data, $I_{\{V 1, V 2\}}(x)(x=1,2,3, \cdots, M)$, as single files, as shown in Fig. 1.

(2) Apply 1D FSV to each row and each column separately and calculate all the $x D M i$ and $x D M$ tot $(x$ is $A, F$ or $G$ ) value for each data. Note: subscripts $v$ and $h$ represent the vertical and horizontal directions respectively.

$$
\begin{aligned}
& x D \operatorname{Di}_{V}(x)=F S V\left(I_{V 1}(x), I_{V 2}(x)\right) \quad x=1,2,3, \cdots, M \\
& x D M i_{H}(y)=F S V\left(I_{H 1}(y), I_{H 2}(y)\right) \quad y=1,2,3, \cdots, N
\end{aligned}
$$

(3) Join all the data and get back to 2D data.

The proposed 2D FSV treats vertical and horizontal parts of the data separately and recombines them at the end. This way is as close as possible to the manner in which engineers would approach the analysis of magnitude-phase data. The magnitude and phase parts are considered separately and then weighted in the process of forming an overall opinion [18].
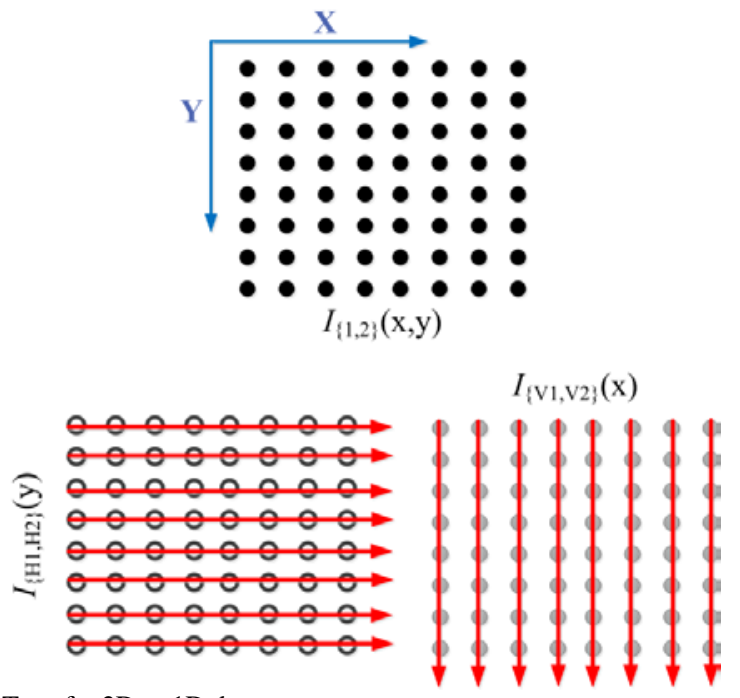

Fig. 1 Transfer 2D to 1D data.

The 1D FSV is separately performed on the vertical and horizontal data and combined on a point-by-point basis through the weighting factors, $K$, according to:

$$
\begin{aligned}
& \operatorname{ADMi}(x, y)=\sqrt{K_{V} \cdot A D M i_{V}(x, y)^{2}+K_{H} \cdot A D M i_{H}(x, y)^{2}} \\
& \operatorname{FDMi}(x, y)=\sqrt{K_{V} \cdot \operatorname{FDMi}_{V}(x, y)^{2}+K_{H} \cdot \operatorname{FDMi}_{H}(x, y)^{2}}
\end{aligned}
$$

where $A D M i$ and $F D M i$ are the combined point-by-point results of 2D data; $A D M i_{\{V, H\}}$ and $F D M i_{\{V, H\}}$ are the 1D FSV results of vertical and horizontal parts of 2D data; the weighting factor $K_{\{V, H\}}$ ranges from 0 to 1 . They are related by following constraint.

$$
K_{V}+K_{H}=1
$$

The calculation of $K_{\{V, H\}}$ in (6) and (7) is adopted by considering that the weight of vertical or horizontal information given in 2D data is proportional to the length of data. In this way, the 1D FSV result can be regarded as the extreme case of 2D data when the value of $M$ or $N$ approaches zero. In that case, the calculation in (3) and (4) is consistent with that of 1D FSV.

$$
\begin{aligned}
K_{V} & =\frac{M}{M+N} \\
K_{H} & =\frac{N}{M+N}
\end{aligned}
$$

where $M$ and $N$ are the length of vertical and horizontal data, respectively.

The GDMi of 2D data comparison combines the ADMi and FDMi without the inclusion of a separate weighting factor, which is in line with 1D FSV approach.

$$
\operatorname{GDMi}(x, y)=\sqrt{\operatorname{ADMi}(x, y)^{2}+\operatorname{FDMi}(x, y)^{2}}
$$

If $G D M_{\{V, H\}}$ is defined as the global point-by-point results of vertical and horizontal data, as outlined in (9), we get (10) by 
substituting (3), (4) and (9) into (8).

$$
\begin{aligned}
\operatorname{GDMi}_{\{V, H\}}(x, y)=\sqrt{A D M i_{\{V, H\}}(x, y)^{2}+F D M i_{\{V, H\}}(x, y)^{2}} & (9) \\
\operatorname{GDMi}(x, y) & =\sqrt{A D M i(x, y)^{2}+F D M i(x, y)^{2}} \\
& =\sqrt{K_{V} \cdot A D M i_{V}(x, y)^{2}+K_{V} \cdot F D M i_{V}(x, y)^{2}+K_{H} \cdot A D M i_{H}(x, y)^{2}+K_{H} \cdot F D M i_{H}(x, y)^{2}} \\
& =\sqrt{K_{V} \cdot G D M i_{V}(x, y)^{2}+K_{H} \cdot G D M i_{H}(x, y)^{2}}
\end{aligned}
$$

Equation (10) indicates the consistency of (3), (4) and (8) in the combination of vertical and horizontal information.

Based on the $x D M i$ results, the $x D M t o t$ is calculated in the same way as 1D FSV.

$$
x D M t o t=\frac{\sum_{i=1}^{M} \sum_{j=1}^{N} x D M i(i, j)}{M \cdot N}, x=\{A, F, G\}
$$

The proposed algorithm may also be adopted easily for the $n \mathrm{D}$ cases. In that case, the equations (3)-(7) are rewritten as

$$
\begin{gathered}
A D M i=\sqrt{\sum_{d=1}^{D} K_{d} \cdot A D M i_{d}{ }^{2}} \\
F D M i=\sqrt{\sum_{d=1}^{D} K_{d} \cdot F D M i_{d}{ }^{2}} \\
\sum_{d=1}^{D} K_{d}=1 \\
K_{d}=\frac{N_{d}}{\sum_{i=1}^{D} N_{i}}
\end{gathered}
$$

where $D$ is the dimensionality of data under comparison, $K_{d}$ is the weighting factor for the $d^{\text {th }}$ dimensional data in the combination of $A D M i$ and FDMi of $n \mathrm{D}$ data, $N_{d}$ is the length of the $d^{\text {th }}$ dimensional data.

After that, the calculation of GDMi values is the same as 2D FSV, as given in equation (8). And the relationship revealed in equation (10) is still valid for the $n \mathrm{D}$ cases. The proof is not presented here.

For the $n \mathrm{D}$ cases, the proposed approach, independently and repeatedly applying 1D FSV in each 'direction', should naturally suited to parallelization, multi-threading and GPU implementation.

\section{Verification of Performance}

\section{A. LIVE Database Test}

As part of the development and verification of the proposed approach, the "LIVE Image Quality Assessment Database Release 2" [19]-[21] (in the next named "LIVE database"), is used to see how different FSV compares with visual assessment. The GDMtot values are computed for the LIVE database which is a publicly available subject-rated image database including "JPEG" (233 images) and "JPEG2000" (227 images). The images in the database were generated by compressing 29 high-resolution 24-bits/pixel RGB color images (typically 768 $x$ 512) using JPEG or JPEG2000 with different compression ratios.

The mean opinion score (MOS) of the database images was obtained by subjective experiment. The testing procedure was as follows: Each of the 20 to 25 observers was shown the images randomly. Observers were asked to provide their perception of quality on a continuous linear scale that was divided into five equal regions marked with adjectives: Bad, Poor, Fair, Good and Excellent. The scale was then converted into a linear scale between 1-100. The general information of the LIVE database is presented in Table I [20].

It is known that FSV method gives quantitative and qualitative assessment regarding the data difference. Their relationship is shown in Table II. To compare the qualitative results with MOS, the six-grade FSV descriptor is piece-wisely converted to quantitative scales, from 0 to 0.6 .

When the images were in RGB color, a Matlab [22] function "rgb2gray" was used to convert the images to gray scale. Both the 2D-FSV-HIT and 2D-FSV-UAq approaches were applied to the LIVE database. The performance evaluations based on Pearson's rank correlation coefficient (PRCC) [23] are outlined in Table III. Both the quantitative and qualitative data are analyzed.

TABLE I (FROM [20])

NUMBER OF IMAGES AND SUBJECTS IN THE LIVE DATABASE

\begin{tabular}{|c|c|c|}
\hline Session & Number of images & Number of subjects \\
\hline JPEG \#1 & 116 & 20 \\
\hline JPEG \#2 & 117 & 20 \\
\hline JPEG2000 \#1 & 116 & 29 \\
\hline JPEG2000 \#2 & 111 & 25 \\
\hline
\end{tabular}

TABLE II

FSV INTERPRETATION SCALE

\begin{tabular}{|c|c|c|}
\hline \multirow{2}{*}{$\begin{array}{c}\text { FSV value } \\
\text { (quantitative) }\end{array}$} & \multicolumn{2}{|c|}{ FSV interpretation (qualitative) } \\
\cline { 2 - 3 } & $\begin{array}{c}\text { Natural language } \\
\text { description }\end{array}$ & $\begin{array}{c}\text { Piece-wise visual } \\
\text { conversion }\end{array}$ \\
\hline $0 \leq \mathrm{y} \leq 0.1$ & Excellent & $\mathrm{y}$ \\
\hline $0.1<\mathrm{y} \leq 0.2$ & Very Good & $\mathrm{y}$ \\
\hline $0.2<\mathrm{y} \leq 0.4$ & Good & $0.2+(\mathrm{y}-0.2) / 2$ \\
\hline $0.4<\mathrm{y} \leq 0.8$ & Fair & $0.3+(\mathrm{y}-0.4) / 4$ \\
\hline $0.8<\mathrm{y} \leq 1.6$ & Poor & $0.4+(\mathrm{y}-0.8) / 8$ \\
\hline $1.6<\mathrm{y} \leq 3.2$ & Very Poor & $0.5+(\mathrm{y}-1.6) / 16$ \\
\hline $\mathrm{y}>3.2$ & & 0.6 \\
\hline
\end{tabular}

TABLE III

CORRELATIONS BETWEEN DifFERENT METHODS

\begin{tabular}{|c|c|c|}
\hline Result Source & Method & PRCC \\
\hline \multirow{2}{*}{$\begin{array}{c}\text { Quantitative FSV result } \\
\text { \& MOS }\end{array}$} & 2D-FSV-HIT vs. 2D-FSV-UAq & 0.89 \\
\cline { 2 - 3 } & 2D-FSV-HIT vs. MOS & -0.94 \\
\cline { 2 - 3 } & 2D-FSV-UAq vs. MOS & -0.83 \\
\hline \multirow{2}{*}{$\begin{array}{c}\text { Qualitative FSV result } \\
\text { \& MOS }\end{array}$} & 2D-FSV-HIT vs. 2D-FSV-UAq & 0.89 \\
\cline { 2 - 3 } & 2D-FSV-HIT vs. MOS & -0.94 \\
\cline { 2 - 3 } & 2D-FSV-UAq vs. MOS & -0.85 \\
\hline
\end{tabular}

The comparison of quantitative results in Table III illustrates that the proposed 2D-FSV-HIT approach shows an improved linear relationship with visual assessment. The minus PRCC 
values are due to the opposite scaling method applied by FSV and MOS. In addition, the two 2D-FSV approaches show significant linear correlation, the difference between them is discussed here.

Comparing with quantitative results, the qualitative outputs of FSV method show better consistency with that of MOS.

The scatter plots of the subjective measurement (MOS) versus the objective predictions (2D-FSV-HIT and 2D-FSV-UAq) are shown in Fig. 2, where each point represents one image under comparison. It is demonstrated that the assessment given by 2D-FSV methods show a linear relation with visual assessment. Particularly, the qualitative results demonstrate a better linear correlation, as shown in Fig. 3, which is in line with Table III.

Further, it is shown that, for the same MOS scale, the dispersion of the 2D-FSV-UAq results in Fig. 2 and Fig. 3 is much wider than that of 2D-FSV-HIT, which leads to the PRCC difference in Table III.

It is noted that a number of points fall on the x-axis in Fig. 2 . This should be attributed to the variability and ambiguity of visual assessment, because the non-existent difference between two physically identical pictures may be visually identified.

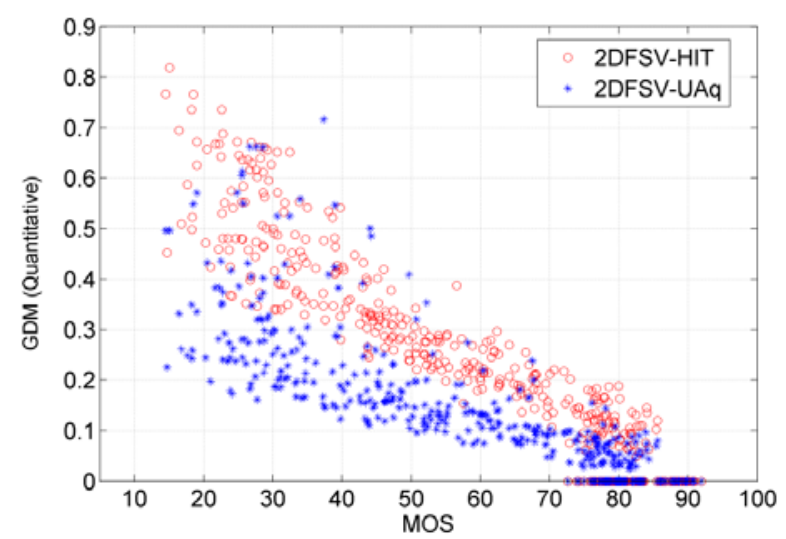

Fig. 2 Scatter plot of MOS and 2D-FSV quantitative results.

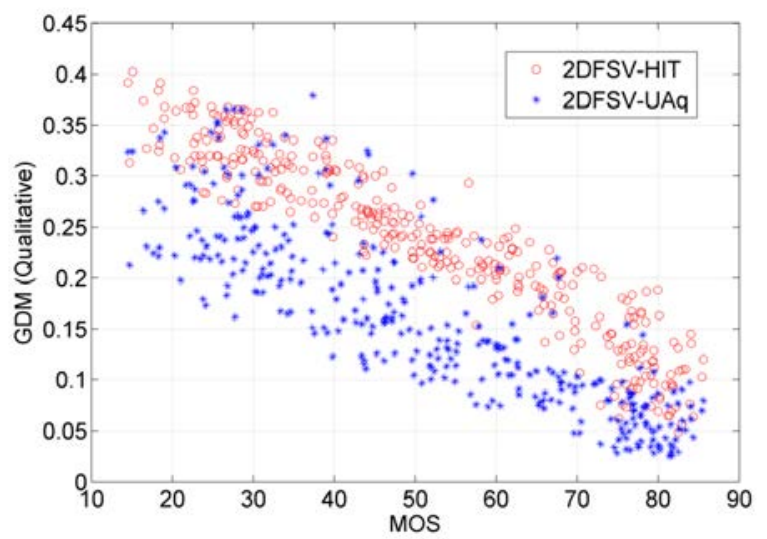

Fig. 3 Scatter plot of MOS and 2D-FSV qualitative results.

\section{B. The Difference between Two FSV 2D Strategies}

It is demonstrated in Fig. 2 and Fig. 3 that the results given by 2D-FSV-HIT and 2D-FSV-UAq are different. This may be caused by the different procedures applied in the calculation of
FSV indicators.

Generally, the 2D-FSV-UAq is developed by exactly following the procedures of 1D FSV and replacing the 1D Fourier Transform by a padded square 2D Fourier Transform. So its results show a better consistency with 1D FSV from the point of mathematics, thereby ensuring the consistence of natural language description of the "quality". While the 2D-FSV-HIT shows advantage from the point of extending to $\mathrm{nD}$ and easy implementation. Further, it shows a better linear correlation with visual assessment, as shown in Table III.

The difference presented by the two methods may be attributed to the different routes of getting the DC, Low- and High-frequency parts of the original data. For the 2D-FSV-HIT method, the decomposition of data is performed in 1D (line and column) and then the decomposed parts are recombined to 2D (labeled as 2*1D). But for the 2D-FSV-UAq method, the decomposition of data is directly performed in 2D. The difference between their filters is compared in Fig. 4.

Fig. 5 compares the filtered components given by the $2 * 1 \mathrm{D}$ and $2 \mathrm{D}$ approaches. It is found that both the approaches could extract corresponding trend and detail information, while the components are slightly different, which leads to the discrepancy of $x D M i$ values in the following calculation.

It should be noted that the combination of $1 \mathrm{D}$ data, e. g. DC part, in Fig. 5 is completed by

$$
D C(x, y)=\sqrt{0.5 * D C_{V}(x, y)^{2}+0.5 * D C_{H}(x, y)^{2}}
$$

where $D C_{V}$ and $D C_{H}$ are the DC part of vertical and horizontal 1D data, respectively. The combinations of Lowand High-frequency components are performed in the same way.

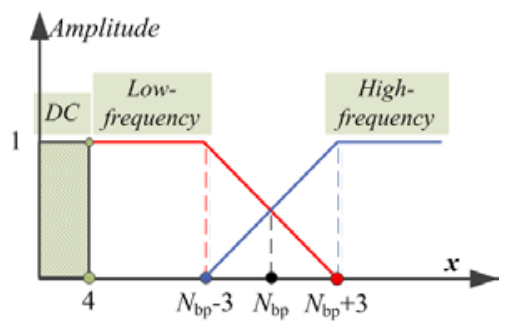

(a) filter used in 1D FSV

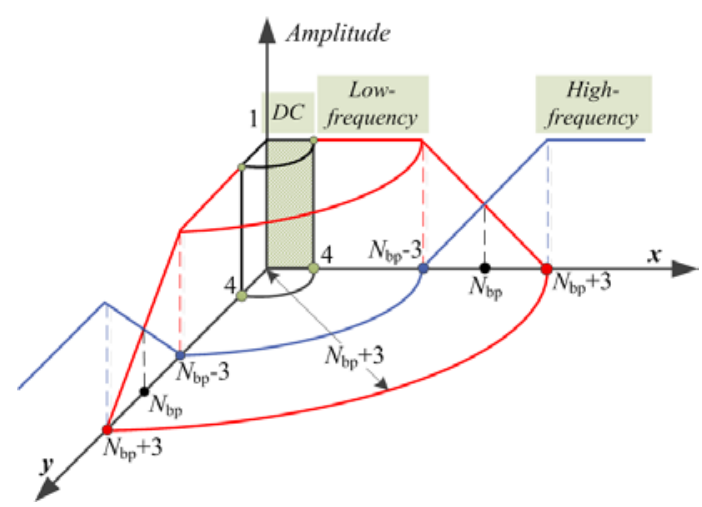

(b) filter used in 2D-FSV-UAq

Fig. 4 Filters defined in the FSV method. 


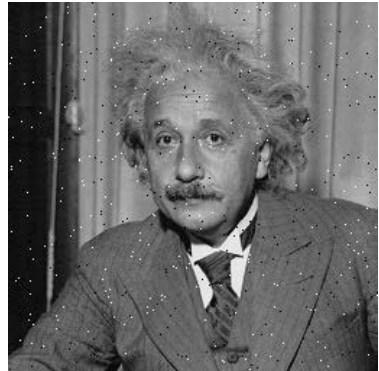

(a) Original Image

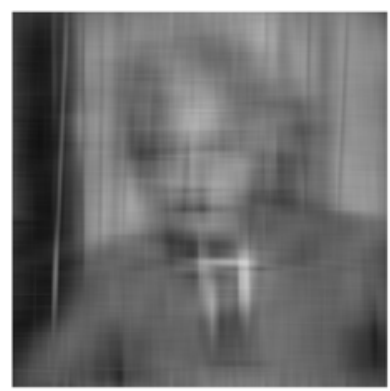

(b1) DC (2*1D-FFT)

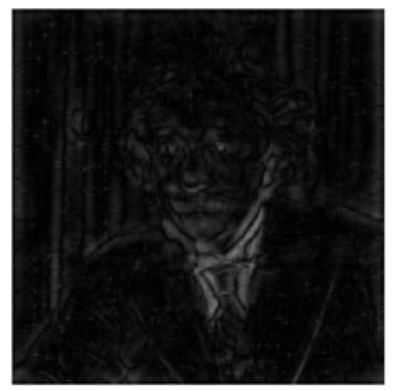

(b2) Low-frequency part (2*1D-FFT)

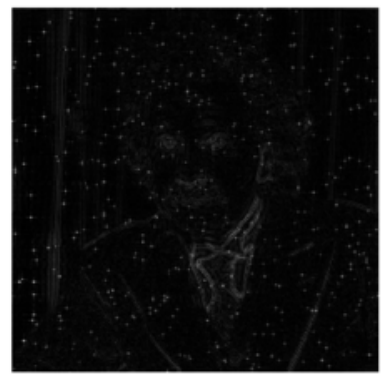

(b3) High-frequency part (2*1D-FFT) Fig. 5 Filtered parts of the original pict

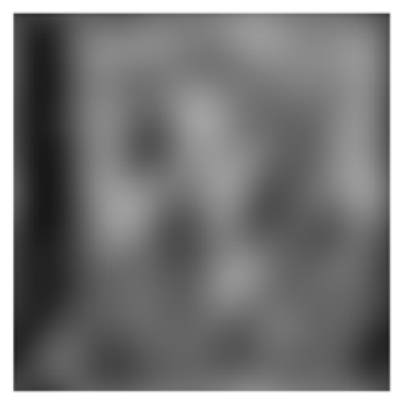

(c1) DC (2D-FFT)

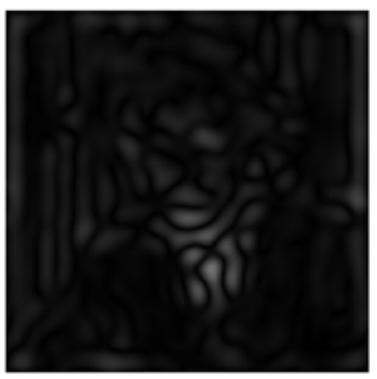

(c2) Low-frequency part (2D-FFT)

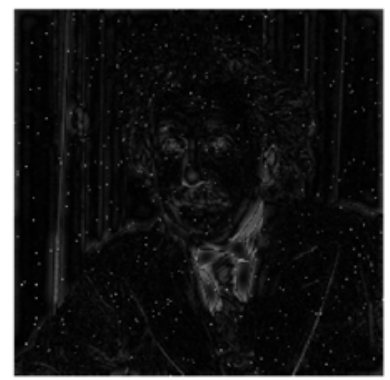

(c3) High-frequency part (2D-FFT) given by different methods.
Actually, it is difficult to define a quantitative relation between the two methods considering the non-linear property of the FSV algorithm. Therefore, to combine the advantages of the 2D-FSV-UAq and 2D-FSV-HIT methods, a coefficient of correction $K_{C}$ is introduced as

$$
\text { GDMtot }=\text { GDMtot }_{\text {HIT }} / K_{C} \text {, }
$$

where the GDMtot and GDMtot ${ }_{\text {HIT }}$ are the corrected and raw results, respectively.

The value of $K_{C}$ is preliminarily discussed based on the LIVE database. Both the methods are applied to the database. Fig. 6 shows their scatter plots of GDMtot values and the linear relationship given in the least-squares sense [24]. Therefore, the value of $K_{C}$ may be drawn from the results. Considering the mathematical consistency of the 2D-FSV-UAq and 1D FSV method, the value of $K_{C}$ is tuned by

$$
K_{C}=\frac{\text { GDMtot }_{H I T}}{\text { GDMtot }_{U A q}} .
$$

where GDMtot $_{U A q}$ is the 2D-FSV-UAq results.

So the value of $K_{C}$ can be preliminarily set to 1.5 based on the available data in Fig. 6.

It should be noted that this scaling constant exists simply to compare the data obtained from the two methods and show that they are similar. It is not a requirement to extend FSV to higher dimensions.

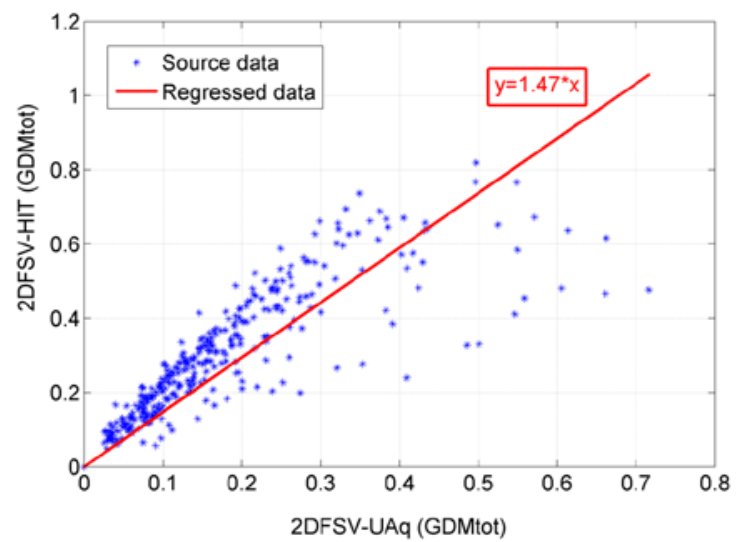

Fig. 6 Scatter plot of 2D-FSV-HIT and 2D-FSV-UAq quantitative results.

\section{EXAMPLE OF IMPLEMENTATION}

To further investigate the proposed 2D-FSV method and demonstrate its operation, a set of electromagnetic simulation results are also compared. The data comes from a reverberation chamber simulation with configurations shown in Fig. 7. Different 2D electric field plots are obtained by changing the stirrer angle $\theta$ or the plane position at which the data was obtained (2800 $\mathrm{mm}$ and $1190 \mathrm{~mm}$ ) through the Transient solver of the CST MWS software [25], as shown in Fig. 7. Fig. 8 presents the 2D electric field data and their corresponding angles and positions are outlined in Table IV.

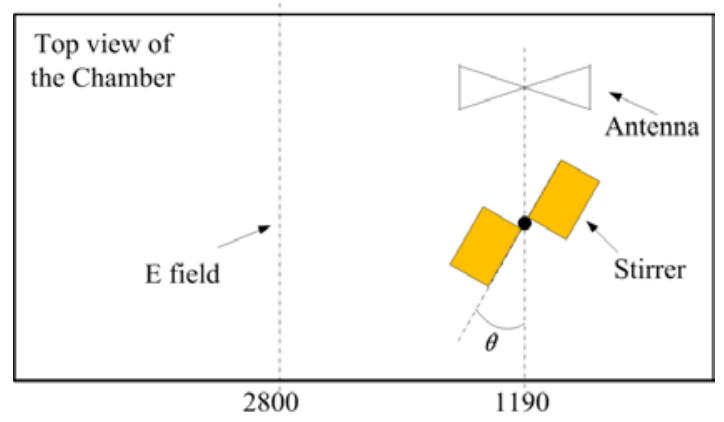

Fig. 7 Top view of the Chamber.

Both 2D-FSV-HIT and 2D-FSV-Uaq were applied to compare the data shown in Fig. 7 and Image-1 was selected as the referencing data. The $x D M$ tot values were also compared in Table IV. It is clear that the outputs of 2D-FSV-HIT are much greater than that of 2D-FSV-UAq. While both the methods 
reveal that, comparing with Image-1, the trend difference (ADMtot) of Image-2 and Image-3 is smaller than feature difference (FDMtot), and the rest of the images show the opposite characteristic.

The scatter plot of GDMtot values given by the two methods are shown in Fig. 9, which suggests a clear linear correlation.
The proportional coefficient given in the least-squares sense is also plotted in Fig. 9, which is in line with coefficient of correction $K_{C}$ in (18). The value 1.56 is close to the predefined value 1.5 .

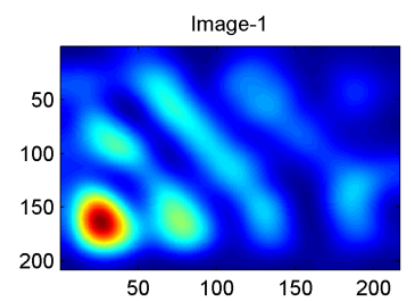

Image-5

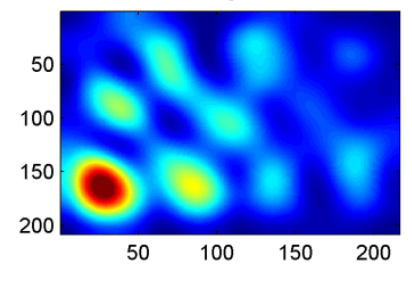

Image-9

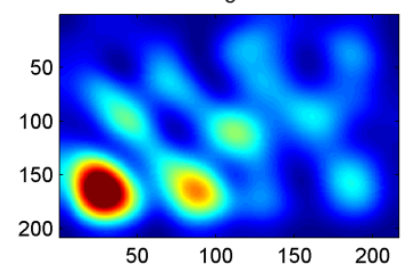

Image-2

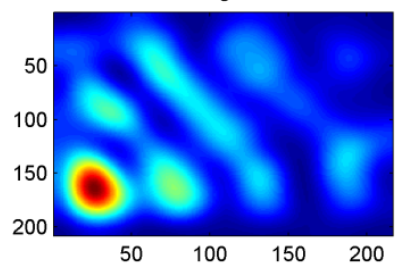

Image-6

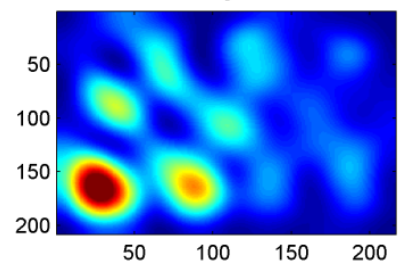

Image-10

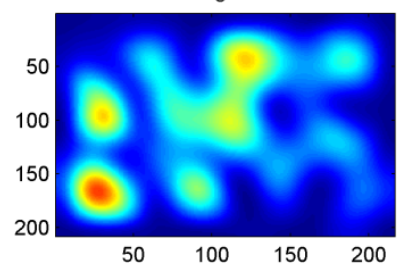

Image-3

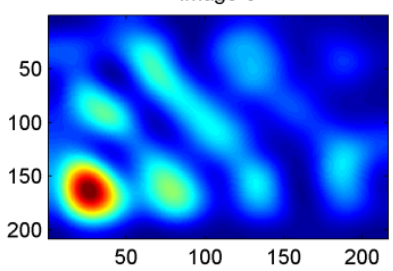

Image-7

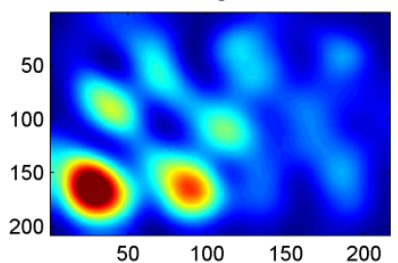

Image-11

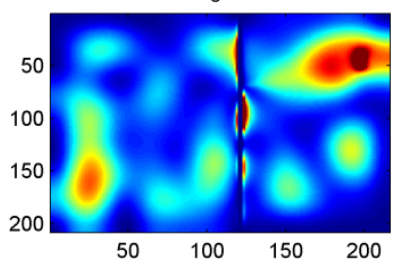

Image-4

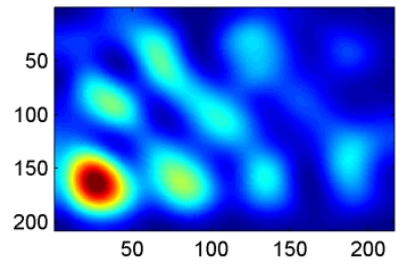

Image-8

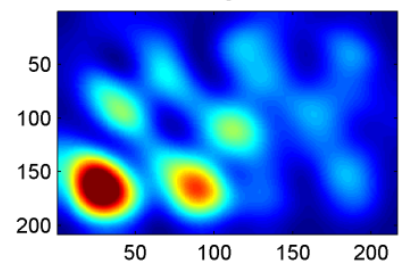

Fig. 8 Electric field images under the same color scale.

TABLE IV

ASSESSMENT RESULTS GIVEN BY DIFFERENT METHODS

\begin{tabular}{|c|c|c|c|c|c|c|c|}
\hline \multirow{2}{*}{$\begin{array}{c}\text { Images } \\
\text { vs. Image-1 }\end{array}$} & \multirow{2}{*}{ Data $(\boldsymbol{\theta})$} & \multicolumn{3}{|c|}{ 2D-FSV-HIT } & \multicolumn{3}{c|}{ 2D-FSV-UAq } \\
\cline { 3 - 8 } & & ADMtot & FDMtot & GDMtot & ADMtot & FDMtot & GDMtot \\
\hline Image-1 & E2800-field (0) & 0 & 0 & 0 & 0 & 0 & 0 \\
\hline Image-2 & E2800-field (2) & 0.1139 & 0.1599 & 0.2112 & 0.0463 & 0.0659 & 0.0891 \\
\hline Image-3 & E2800-field (5) & 0.2501 & 0.2760 & 0.4006 & 0.1106 & 0.1166 & 0.1791 \\
\hline Image-4 & E2800-field (10) & 0.4416 & 0.4389 & 0.6680 & 0.2099 & 0.2043 & 0.3280 \\
\hline Image-5 & E2800-field (15) & 0.5630 & 0.5463 & 0.8476 & 0.3071 & 0.2752 & 0.4619 \\
\hline Image-6 & E2800-field (20) & 0.6490 & 0.5768 & 0.9410 & 0.3979 & 0.3250 & 0.5764 \\
\hline Image-7 & E2800-field (25) & 0.7169 & 0.6268 & 1.0360 & 0.4701 & 0.3660 & 0.6694 \\
\hline Image-8 & E2800-field (30) & 0.7266 & 0.6399 & 1.0558 & 0.5021 & 0.3733 & 0.7040 \\
\hline Image-9 & E2800-field (35) & 0.7009 & 0.6551 & 1.0505 & 0.4837 & 0.3486 & 0.6678 \\
\hline Image-10 & E2800-field (90) & 0.9180 & 0.7256 & 1.2720 & 0.6050 & 0.4510 & 0.8409 \\
\hline Image-11 & E1190-field (25) & 1.7927 & 1.2477 & 2.4302 & 1.0320 & 0.8919 & 1.5928 \\
\hline
\end{tabular}




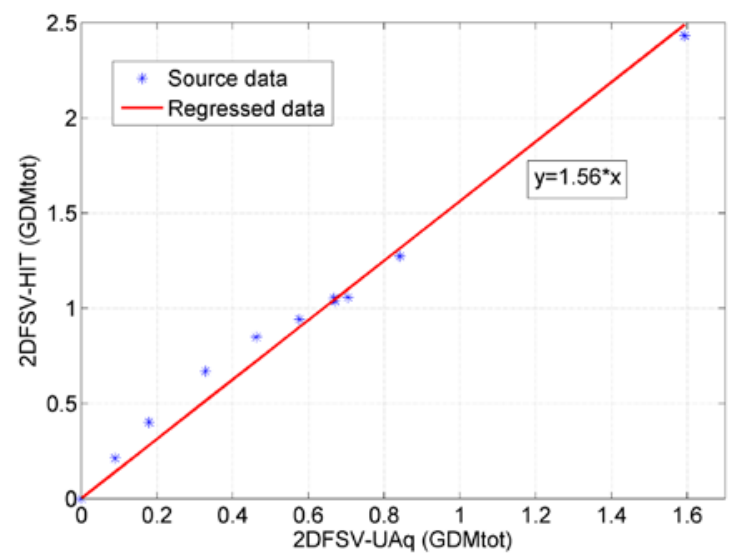

Fig. 9 Scatter plot of 2D-FSV-HIT and 2D-FSV-UAq quantitative results.

\section{CONCLUSION}

In this paper, a simple numerical $n \mathrm{D}$ data validation approach, 2D-FSV-HIT, is proposed based on the 1D-FSV method and evaluated with a subject-rated image database, the LIVE database. The advantage of the proposed algorithm is that it can be easily extended to higher dimensional data comparison based on the well-established 1D FSV method. Further, the 2D-FSV-HIT method allows data with different number of points in each direction to be compared.

It is demonstrated that the proposed approach shows a better linear correlation with visual assessment results than the 2D Fourier Transform based 2D-FSV-UAq method. The disagreement between outputs of the two 2D FSV approaches is attributed to the different filters that applied in the decomposition process of 2D data. Therefore, a coefficient of correction, $K_{C}$, was introduced to combine the advantages of 2D-FSV-HIT and 2D-FSV-UAq and keep the consistency of natural language description with 1D FSV method. And the value of $K_{C}$ is preliminarily set to 1.5 based on the LIVE database comparison, which is further verified by an example of electromagnetic simulation.

Higher dimensional extension of the 2D-FSV-HIT method is also investigated. However, taking into account the difficulty in obtaining visual assessment results, the verification of the extension strategy is a topic of on-going research.

\section{ACKNOWLEDGMENT}

The authors would like to express their gratitude to Dr. Archambeault Bruce for providing the reverberation chamber simulation model and data.

\section{REFERENCES}

[1] B. Archambeault, J. Diepenbrock, "Quantifying the quality of agreement between simulation and validation data for multiple data sets," 2010 IEEE International Symposium on Electromagnetic Compatibility, 2010, pp.722-725.

[2] S. Pan, H. Wang, J. Fan, "Comparison among Different Via Models Based on Feature Selective Validation Technique," IEEE International Symposium on Electromagnetic Compatibility, Austin, 2009, pp. 264-267.
[3] A. P. Duffy, A. J. M. Martin, A. Orlandi, G. Antonini, T. M. Benson, and M. S. Woolfson, "Feature selective validation (FSV) for validation of computational electromagnetics (CEM). Part I - The FSV method," IEEE Transactions on Electromagnetic Compatibility, vol. 48, pp. 449-459, Aug 2006.

[4] A. Orlandi, A. P. Duffy, B. Archambeault, G. Antonini, D. E. Coleby, and S. Connor, "Feature selective validation (FSV) for validation of computational electromagnetics (CEM). Part II - Assessment of FSV performance," IEEE Transactions on Electromagnetic Compatibility, vol. 48, pp. 460-467, Aug 2006.

[5] A. J. M. Martin, "Quantitative data validation," Ph. D. dissertation Ph. D. dissertation, De Montfort Univ., Leicester, U.K., 1999.

[6] "IEEE Standard for Validation of Computational Electromagnetics Computer Modeling and Simulations," IEEE STD 1597.1-2008, pp. c1-41, 2008.

[7] "IEEE Recommended Practice for Validation of Computational Electromagnetics Computer Modeling and Simulations," IEEE Std 1597.2-2010, pp. 1-124, 2011.

[8] D. E. Coleby and A. P. Duffy, "A visual interpretation rating scale for validation of numerical models," Compel-the International Journal for Computation and Mathematics in Electrical and Electronic Engineering, vol. 24, pp. 1078-1092, 2005.

[9] B. Archambeault, S. Connor, A. Duffy, and Ieee, "Comparing FSV and human responses to data comparisons," in EMC 2005: IEEE International Symposium on Electromagnetic Compatibility, Vols 1-3, Proceedings, ed, 2005, pp. 284-289.

[10] R. Jauregui, G. Zhang, J. Rojas-Mora, O. Ventosa, F. Silva, A. P. Duffy, et al., "Analyzing Transient Phenomena in the Time Domain Using the Feature Selective Validation (FSV) Method," IEEE Transactions on Electromagnetic Compatibility, vol. 56, pp. 825-834, 2014.

[11] G. Zhang, A. P. Duffy, H. Sasse, L. Wang, and R. Jauregui, "Improvement in the Definition of ODM for FSV," IEEE Transactions on Electromagnetic Compatibility, vol. PP, pp. 1-7, 2012.

[12] A. Duffy, A. Orlandi, and H. Sasse, "Offset difference measure enhancement for the feature-selective validation method," IEEE Transactions on Electromagnetic Compatibility, vol. 50, pp. 413-415, 2008.

[13] A. Orlandi, G. Antonini, C. Ritota, and A. P. Duffy, "Enhancing Feature Selective Validation (FSV) interpretation of EMC/SI results with grade-spread," in IEEE International Symposium on Electromagnetic Compatibility, Portland, 2006, pp. 362-367.

[14] A. Orlandi, G. Antonini, C. Polisini, A. Duffy, and H. Sasse, "Progress in the Development of a 2D Feature Selective Validation (FSV) Method," in 2008 Ieee International Symposium on Electromagnetic Compatibility, Vols 1-3, ed, 2008, pp. 818-823.

[15] A. J. M. Martin, A. R. Ruddle, and A. P. Duffy, " (2D) Comparison of measured and computed local electric field distributions due to vehicle-mounted antennas using 2D feature selective validation," in 2005: IEEE International Symposium on Electromagnetic Compatibility, Vols 1-3, Proceedings, ed, 2005, pp. 290-295.

[16] B. Archambeault, A. Duffy, H. Sasse, X. K. Li, M. Scase, M. Shafiullah, et al., "Challenges in developing a multidimensional feature Selective Validation implementation," in 2010 IEEE International Symposium on Electromagnetic Compatibility, EMC 2010, July 25, 2010 - July 30, 2010, Fort Lauderdale, FL, United states, 2010, pp. 726-731.

[17] A. L. Drozd, B. Archambeault, A. Duffy, and I. Kasperovich, "Development of next generation FSV tools and standards," in Electromagnetic Compatibility (EMC), 2012 IEEE International Symposium on, 2012, pp. 674-678.

[18] D. E. Coleby and A. P. Duffy, "Analysis of techniques to compare complex data sets," Compel-the International Journal for Computation and Mathematics in Electrical and Electronic Engineering, vol. 21, pp. 540-553, 2002.

[19] H.R. Sheikh, Z.Wang, L. Cormack and A.C. Bovik, "LIVE Image Quality Assessment Database Release 2", available at http://live.ece.utexas.edu/research/quality.

[20] H.R. Sheikh, M.F. Sabir and A.C. Bovik, "A statistical evaluation of recent full reference image quality assessment algorithms", IEEE Transactions on Image Processing, vol. 15, no. 11, pp. 3440-3451, Nov. 2006. 
[21] Z. Wang, A.C. Bovik, H.R. Sheikh and E.P. Simoncelli, "Image quality assessment: from error visibility to structural similarity," IEEE Transactions on Image Processing, vol.13, no.4, pp. 600- 612, April 2004.

[22] MathWorks, Matlab R2013a, available at http://www.mathworks.com

[23] M. Hollander, D. A. Wolfe, and E. Chicken, Nonparametric statistical methods: John Wiley \& Sons, 2013.

[24] N. R. Draper, H. Smith, and E. Pownell, Applied regression analysis vol. 3: Wiley New York, 1966.

[25] Computer Simulation Technology, CST Studio 2013, available at www.cst.com.

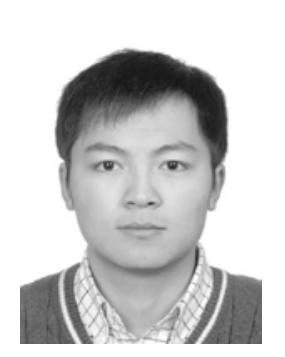

Gang Zhang received the B.Sc. in electrical engineering from China University of Petroleum, Dongying, China, in 2007, and the M.Sc. and Ph.D. degrees in electrical engineering from Harbin Institute of Technology (HIT), Harbin, China, in 2009 and 2014, respectively.

He is now with the Faults Online Monitoring and Diagnosis Laboratory at Harbin Institute of Technology. His research interests include analysis of electromagnetic compatibility, electromagnetic simulation, and the validation of CEM.

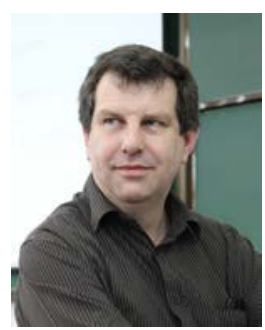

Alistair P. Duffy (M'93-SM'04-F'15) was born in Ripon, U.K., in 1966. He received the B.Eng. (Hons.) degree in electrical and electronic engineering and the M. Eng. degree from the University College, Cardiff, U.K., in 1988 and 1989, respectively. He received the Ph.D. degree from Nottingham University, Nottingham, U.K., in 1993 for his work on experimental validation of numerical modeling and the MBA from the Open University in 2003.

He is currently a Professor in electromagnetics at De Montfort University, Leicester, U.K, and a Guest Professor at Harbin Institute of Technology, Harbin, China. He is the author of over 150 articles published in journals and presented at international symposia. His research interests include CEM validation, communications cabling, and technology management.

Dr. Duffy is a Fellow of the IEEE and of the Institution of Engineering and Technology (IET) and a Member of the International Compumag Society and the Applied Computational Electromagnetics Society. He is a past member of the IEEE EMC Society Board of Directors, and Associate Editor of the IEEE Transactions on EMC, the EMC Society's Global Symposium Coordinator and is the Chair of the IEEE EMC Society Standards Development and Education Committee.

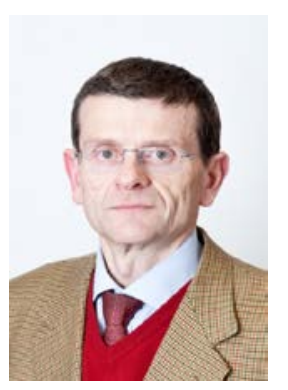

Antonio Orlandi (M'90-SM'97-F'07) was born in Milan, Italy in 1963. He received the Laurea degree in Electrical Engineering from the University of Rome "La Sapienza", Italy, in 1988 and the Ph.D. degree in Biomedical engineering from the University “Campus Biomedico”, Italy, in 2012.

He is currently Full Professor and Chair of the UAq EMC Laboratory, University of L'Aquila, L'Aquila, Italy. Author of more than 300 technical papers, he has published in the field of electromagnetic compatibility in lightning protection systems and power drive systems. Current research interests are in the field of numerical methods and modeling techniques to approach signal/power integrity, EMC/EMI issues in high speed digital systems.

Dr. Orlandi is currently Editor in Chief of the IEEE TRANSACTIONS ON ELECTROMAGNETIC COMPATIBILITY, member of the "Education", TC-9 "Computational Electromagnetics" and Past Chairman of the TC-10 "Signal Integrity" Committees of the IEEE EMC Society. From 1996 to 2000, and from 2010 up today has been Associate Editor of the IEEE TRANSACTIONS ON ELECTROMAGNETIC COMPATIBILITY, from 2001 to 2006 served as Associate Editor of the IEEE TRANSACTIONS ON MOBILE COMPUTING and from 1999 to the end of the Symposium was Chairman of the TC-5 "Signal Integrity" Technical Committee of the International Zurich Symposium and Technical Exhibition on EMC.

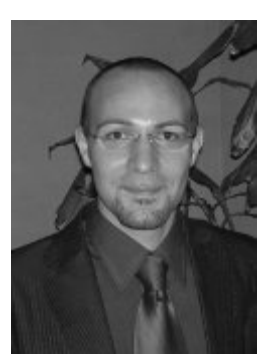

Danilo Di Febo was born in Atri (TE), Italy in 1980. $\mathrm{He}$ received the Laurea degree in Electronic Engineering in 2009 and the PhD degree in Electrical and Information Engineering in 2013, both from the University of L'Aquila, L'Aquila, Italy.

$\mathrm{He}$ is now with the UAq EMC Laboratory at University of L'Aquila and his activities are focused on the development of the Feature Selective Validation (FSV) technique, modeling and analysis of SI/EMC problem in IT equipments.

Lixin Wang received the B.S. degree in electrical engineering from Nankai

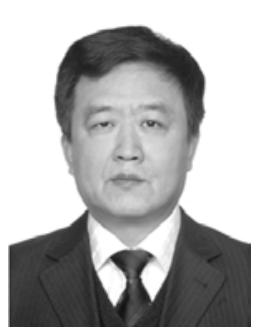
University, Tianjin, China, in 1988, and the M.S. and D.Sc. degrees in electrical engineering from Harbin Institute of Technology (HIT), Harbin, China, in 1991 and 1999, respectively.

He is currently a Professor of Power Electronic and Electric Drives at the HIT. He conducts research with Faults Online Monitoring and Diagnosis Laboratory, HIT, on a wide variety of topics including electromagnetic compatibility at the electronic system level, aircraft electromechanical fault diagnosis expert system and prediction and health management (PHM) of Li-ion battery.

Hugh Sasse graduated from the University of York, York, U.K., in 1985, and

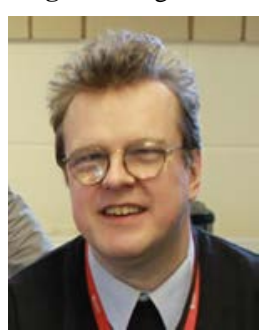
received the Ph.D. degree from De Montfort University, Leicester, U.K., in 2010.

His main interests are computer modeling of novel antenna structures and other communication channel components, with an emphasis on optimization of such structures. 